\title{
Sentinel seroprevalence of SARS-CoV-2 in Gauteng Province, South Africa, August - October 2020
}

\author{
J A George, ${ }^{1}$ MB BCh, DTM\&H, MSc (Immunol), PhD, FC Path (SA); S Khoza, ${ }^{1}$ BSc, MB BCh, MMed (Chem Path), FC Path (SA); \\ E Mayne, ${ }^{2} \mathrm{MB}$ BCh, MMed (Haem), FC Path (SA); S Dlamini, ${ }^{1}$ BSc, MB BCh, FC Path (SA); N Kone, ${ }^{1}$ MB BCh, FC Path (SA); \\ W Jassat, ${ }^{3} \mathrm{MB}$ BCh, MMed (PH); K Chetty, ${ }^{4} \mathrm{MB}$ BCh, MMed (PH); C M Centner, ${ }^{5}$ MB ChB, MSc, MMed, FC Path (SA) Micro; \\ T Pillay, ${ }^{1} \mathrm{MB}$ ChB, FC Path (SA); M Maphayi, ${ }^{1} \mathrm{MB}$ BCh, MMed (Chem Path), FC Path (SA); \\ D Mabuza, ${ }^{1}$ MB BCh, MMed (Chem Path), FC Path (SA); I Maposa, ${ }^{6}$ BSc, BSc Hons, MSc, PhD; N Cassim, ${ }^{7}$ BTech, MPH \\ ${ }^{1}$ Department of Chemical Pathology, Faculty of Health Sciences, University of the Witwatersrand and National Health Laboratory Service, \\ Johannesburg, South Africa \\ 2 Department of Immunology, Faculty of Health Sciences, University of the Witwatersrand and National Health Laboratory Service, Johannesburg, \\ South Africa \\ ${ }^{3}$ Division of Public Health Surveillance and Response, National Institute for Communicable Disease of the National Health Laboratory Service, \\ Johannesburg, South Africa \\ ${ }^{4}$ National Health Laboratory Service, Johannesburg, South Africa \\ ${ }^{5}$ Division of Medical Microbiology, Groote Schuur Hospital, National Health Laboratory Service and Faculty of Health Sciences, University of \\ Cape Town, South Africa \\ ${ }^{6}$ Division of Epidemiology and Biostatistics, School of Public Health, Faculty of Health Sciences, University of the Witwatersrand, Johannesburg, \\ South Africa \\ ${ }^{7}$ Department of Molecular Medicine and Haematology, Faculty of Health Sciences, University of the Witwatersrand and National Health \\ Laboratory Service, Johannesburg, South Africa
}

Corresponding author: J A George (jaya.george@wits.ac.za)

\begin{abstract}
Background. Estimates of prevalence of anti-SARS-CoV-2 antibody positivity (seroprevalence) for tracking the COVID-19 epidemic are lacking for most African countries.

Objectives. To determine the prevalence of antibodies against SARS-CoV-2 in a sentinel cohort of patient samples received for routine testing at tertiary laboratories in Johannesburg, South Africa.

Methods. This sentinel study was conducted using remnant serum samples received at three National Health Laboratory Service laboratories in the City of Johannesburg (CoJ) district. Collection was from 1 August to 31 October 2020. We extracted accompanying laboratory results for glycated haemoglobin (HbAlc), creatinine, HIV, viral load and CD4 T-cell count. An anti-SARS-CoV-2 targeting the nucleocapsid (N) protein of the coronavirus with higher affinity for IgM and IgG antibodies was used. We reported crude as well as population-weighted and test-adjusted seroprevalence. Multivariate logistic regression analysis was used to determine whether age, sex, HIV and diabetic status were associated with increased risk for seropositivity.

Results. A total of 6477 samples were analysed, the majority ( $n=5$ 290) from the CoJ region. After excluding samples with no age or sex stated, the model population-weighted and test-adjusted seroprevalence for the CoJ ( $n=4393$ ) was $27.0 \%$ ( $95 \%$ confidence interval (CI) 25.4 - 28.6). Seroprevalence was highest in those aged 45 - 49 years $(29.8 \%$; $95 \%$ CI 25.5 - 35.0) and in those from the most densely populated areas of the CoJ. Risk for seropositivity was highest in those aged 18 - 49 years (adjusted odds ratio (aOR) 1.52; $95 \%$ CI 1.13 - 2.13; $p=0.0005)$ and in samples from diabetics (aOR 1.36 ; $95 \%$ CI $1.13-1.63 ; p=0.001$ ).

Conclusions. Our study conducted between the first and second waves of the pandemic shows high levels of current infection among patients attending public health facilities in Gauteng Province.
\end{abstract}

S Afr Med J 2021;111(11):1078-1083. https://doi.org/10.7196/SAMJ.2021.v111i11.15669

SARS-CoV-2 infection is responsible for the global COVID-19 outbreak. $^{[1]}$ The World Health Organization declared COVID-19 a global pandemic on 11 March 2020. ${ }^{[2]}$ In South Africa (SA), the first case of COVID-19 was reported on 5 March 2020. ${ }^{[3,4]}$ To limit the spread of the disease, the government introduced a national lockdown with a five-level COVID-19 alert system, starting with alert level 5 on 27 March 2020. This containment measure delayed the peak of COVID-19 disease; ${ }^{[4]}$ however, as restrictions eased, most of SA experienced its first peak in July 2020. ${ }^{[5]}$

As of the end of June 2021, SA had $>1.8$ million laboratoryconfirmed COVID-19 cases. ${ }^{[6]}$ Confirmation of cases is by amplification and detection of SARS-CoV-2 RNA in respiratory samples, using real-time reverse transcription polymerase chain reaction (RT-PCR) assays. PCR testing is mostly offered to both symptomatic persons and contacts (defined as asymptomatic persons who have had close contact with a confirmed COVID-19 case). ${ }^{[7]}$ This testing strategy precludes the ability to estimate the true burden of disease and importantly cannot detect disease in recovered individuals.

Seroprevalence surveys may provide a better understanding of the epidemiology of COVID-19 than PCR testing. Testing to detect anti-SARS-CoV-2 antibodies is able to assess the true number of infections more accurately. The majority of these antibody studies from around the world conducted early in the epidemic revealed a pooled SARS-CoV-2 seroprevalence of $3.38 \%$ ( $95 \%$ confidence 
interval (CI) $3.05-3.72) .{ }^{[8]}$ There is a paucity of similar studies in Africa ${ }^{[9,10]}$ A study from Cape Town that examined the seroprevalence in a sentinel survey of public sector patients found high seropositivity rates, ranging from $31 \%$ to $46 \%$ in different populations. This study was conducted between July and August 2020, after the peak of the first wave in Cape Town, and utilised convenience sampling. The majority of the patients sampled were attending antenatal or HIV clinical care centres and were therefore overwhelmingly female and adult. ${ }^{[1]}$

SA, the country most severely affected on the African continent, has a heavy burden of communicable diseases (especially HIV and tuberculosis) as well as non-communicable diseases such as diabetes mellitus (DM), hypertension, cancers and chronic kidney disease. ${ }^{[12]}$ These may all predispose to severe COVID-19 disease. ${ }^{[13]}$

\section{Objectives}

To estimate the seroprevalence of anti-SARS-CoV-2 antibodies in patients visiting public healthcare centres in Gauteng Province, SA.

\section{Methods}

\section{Ethics approval}

The study was approved by the University of the Witwatersrand Human Ethics Committee (ref. no. M2008105).

\section{Study design}

This was a cross-sectional sentinel study conducted using remnant serum samples received at three National Health Laboratory Service laboratories situated in the City of Johannesburg (CoJ) metropolitan area. These laboratories receive samples from surrounding public sector primary healthcare (PHC) facilities and hospitals.

\section{Sample selection}

We selected remnant samples received at the chemistry departments at Charlotte Maxeke Johannesburg Academic Hospital, Chris Hani Baragwanath Academic Hospital and Helen Joseph Hospital for routine chemistry tests. Samples of adequate volume from hospital outpatient departments and PHC facilities, received from August to October 2020, were selected. Accompanying RT-PCR results for SARS-CoV-2, glycated haemoglobin (HbAlc), creatinine, HIV, viral load and CD4 T-cell count were included. These were linked to demographic information extracted from the Laboratory Information System. Specimens from individual patients were tested only once.

\section{Laboratory methods}

Anti-SARS-CoV-2 antibody testing was performed on the Roche Cobas e604 analyser utilising the Roche Elecsys SARS-CoV-2 chemiluminescent assay (both Roche Diagnostics, Germany). The assay measures total antibodies targeting the nucleocapsid $(\mathrm{N})$ protein of the coronavirus, with higher affinity for IgM and IgG antibodies. Prior to this survey, the assay was extensively validated utilising a well-characterised selection of positive and negative samples $(N=434)$, using local RT-PCR-positive symptomatic individuals and stored negative sera taken prior to December 2019. In summary, this revealed a high cumulative specificity compared with PCR testing of $100 \%$ and a sensitivity which varied from $47.6 \%$ in patients prior to day 7 post diagnosis to $90 \%$ after 30 days in patients with PCRconfirmed SARS-CoV-2 infection. ${ }^{[14]}$

\section{Data preparation}

Age categories reported in the 2020 census population estimates were used as follows: $0-4,5-9,10-14,15-19,20-24,25-29$, 30 - 34, 35 - 39, 40 - 44, $45-49,50-54,55-59,60-64,65-69$,
70 - 74, 75 - 79 and $\geq 80$ years. Data without an age or gender value were classified as unknown. Patients were identified as HIV-infected if either an HIV viral load or a CD4 T-cell count was recorded or if samples came from an antiretroviral clinic or HIV ward. Patients were classified as diabetic if the sample came from a diabetes clinic and/or the HbAlc was $\geq 6.5 \%$. We divided HIV-infected patients by $\mathrm{CD} 4 \mathrm{~T}$-cell count as below the normal reference range ( $\leq 500$ cells $/ \mu \mathrm{L})$ or within the normal reference range $(>500$ cells $/ \mu \mathrm{L})$. A CD4 T-cell count $>500$ cells $/ \mu \mathrm{L}$ was classified as immune reconstitution. The HIV viral load data were classified as $<1000$ copies/mL (virological suppression) and $\geq 1000$ copies $/ \mathrm{mL}$ (detectable viraemia). Based on whether the health facility description included the word 'hospital', collected data were classified as either 'hospital' or 'other' (facility type). All data received as text were converted (coded) to numbers.

\section{Statistical analysis}

Data were captured on an Excel 365 spreadsheet (Microsoft, USA) and analysis was performed using Stata 15 SE statistical software (StataCorp, USA). We reported the number of patients by sex, age category, month and geographical area (health district or regions for the CoJ). We reported the number of patients with a positive SARS$\mathrm{CoV}-2 \mathrm{~N}$-protein IgG result to determine the crude seroprevalence with the $95 \%$ CI indicated. We reported the same data by facility and ward type (Fig. 1A). Furthermore, to improve precision and accuracy of our estimates given a non-random sample, we used a Bayesian logistic multilevel regression model with post-stratification weighting (MRP) that included sex as a fixed effect, and age and region as random effects. The multilevel regression and poststratification models assume heterogeneity in the prevalence of seropositivity in the different regions of the CoJ. The analysis uses the sex-age distribution in each of the CoJ regions as weights. After estimating population-weighted prevalence, we went further to adjust for the assay uncertainty using the multilevel regression and post-stratification model, assuming that the observed prevalence is a function of assay sensitivity and specificity (more details in the supplementary material available at http://samj.org.za/public/ sup/15669.pdf). For this analysis we used a Roche SARS-CoV-2 antibody assay performance of $93 \%$ sensitivity and $99 \%$ specificity based on assay evaluations. ${ }^{[15,16]}$ In seropositivity prevalence analysis, the sensitivity and specificity of the assay are also known to be a source of variation and uncertainty in the estimates. The rjags package (R Foundation for Statistical Computing, Austria) was used to fit the MRP models, and estimates were reported with associated credible intervals. Jags uses Markov Chain Monte Carlo (MCMC) to generate a sequence of dependent samples from the posterior distribution of the parameters. We reported the population-weighted and test performance-adjusted SARS-CoV-2 N-protein IgG seroprevalence. The model used SA Census 2011 population estimates for the CoJ for administration regions A - G by sex and age category.

We compared $\mathrm{HbAlc}$, viral load and $\mathrm{CD} 4$ counts between seropositive and seronegative samples. The Wilcoxon rank sum test was used to assess whether the difference in median values was significant. Multivariable logistic regression analysis was used to determine whether age, gender, HIV and diabetic status were associated with increased risk for seropositivity. Age was categorised as $<18,18-49$ and $>49$ years. We reported sex as male and female.

COVID-19 hospitalisations were extracted from DATCOV, a hospital surveillance system. The DATCOV system was used by hospitals for data elements related to admissions and in-hospital deaths. For the August - October 2020 period, we received the numbers of admissions and in-hospital deaths for the CoJ. 


\section{Results}

Supplementary Table S1 (http://samj.org.za/public/sup/15669.pdf) shows the patient characteristics and crude seroprevalence. A total of 6477 samples were analysed, of which the majority were collected in September 2020 ( $n=3347 / 6477 ; 53.7 \%)$. Of all the samples, 5290 (81.7\%) were from the CoJ district, and 3234 (49.9\%) were from females; 201 (3.1\%) were from children aged $\geq 4$ years, and 1091 patients did not have an age stated on the laboratory request form. Crude seroprevalence was highest in August and lowest in September. While slightly more samples were from patients attending hospital than PHC facilities, the crude seroprevalence was remarkably similar for both $(27.8 \%$; $95 \%$ CI 26.3 - 29.3 v. $27.8 \%$; $95 \%$ CI 26.3 - 29.5, respectively).

The crude seroprevalence in HIV-positive cases was 26.9\% (95\% CI 25.2 - 28.7), and in diabetics 32.3\% (95\% CI 29.1 - 35.7); 299 samples were from women attending antenatal clinics, and for these the crude seroprevalence was $30.8 \%$ (95\% CI $25.6-36.3$ ) (Supplementary Table S2, http://samj.org.za/public/sup/15669.pdf).

To calculate the age- and test-adjusted seroprevalence for the CoJ, we excluded all samples for which there was no age or gender, giving a final number of 4393 samples (Fig. 1A).

SARS-CoV-2 N-protein IgG seroprevalence was calculated between August and October 2020, which was between the two waves in Gauteng (Fig. 1B). The number of SARS-CoV-2 cases peaked at 104663 in wave 1 in July 2020 (Fig. 1B). Similarly, there were 87106 SARS-CoV-2 cases in January 2021 during wave 2.

Table 1 shows the adjusted seroprevalence. Adjusted seroprevalence was higher in males, $(30.1 \%$; $95 \%$ CI 26.5 - 33.2) than in females (23.5\%; $95 \%$ CI 19.4 - 26.7). Seropositivity was $>20 \%$ for all age groups. Seroprevalence was highest in those aged $45-49$ years and lowest in those aged $\leq 4$ years. By subdistrict, seropositivity was highest in administrative regions $\mathrm{E}$ and $\mathrm{F}$, which represent the high-density areas of Alexandra, Wynberg, Sandton, Orange Grove, Houghton, Inner City and Johannesburg South (Fig. 2).

\section{Trend analysis}

Deaths were assessed as a percentage of hospital admissions, and these dropped slightly from $21.2 \%$ to $14.1 \%$ over the study period. At the same time, the crude seroprevalence for the CoJ dropped slightly from $31.3 \%$ to $26.7 \%$ (Table 2).

\section{Logistic regression analysis}

We carried out multivariable logistic regression analysis to determine whether age, HIV status or DM increased risk for seropositivity (Fig. 3). The risk of seropositivity was similar for males and females (adjusted odds ratio (aOR) $0.91 ; 95 \%$ CI $0.81-1.04 ; p=0.19$ ). Patients aged 18 - 49 years were significantly more likely to be COVID19 -seropositive than the $<18$ years age group, with an OR of 1.52 (95\% CI $1.13-2.13 ; p=0.67$ ). Diabetics had an increased risk for seropositivity (aOR 1.36; 95\% CI 1.13 - 1.63; $p=0.001$ ) compared with those who were not diagnosed with DM.

Finally, we compared HbAlc levels, CD4 T-cell counts and viral load in seropositive and seronegative samples (Supplementary Table S3, http://samj.org.za/public/sup/15669.pdf). We showed that $\mathrm{HbAlc}$ was significantly higher in patients who were seropositive for COVID-19 compared with those who tested negative (8.2\%; 95\% CI 6.8 - 11.0 v. $7.5 \%$; $95 \%$ CI 6.2 - 9.4, respectively; $p=0.02$ ). Median (interquartile range) $\mathrm{CD} 4 \mathrm{~T}$-cell counts were higher in seropositive patients than in those who were seronegative (625 (396 - 653) cells/ $\mu \mathrm{L}$ v. $576(302$ - 641) cells/ $\mu \mathrm{L}$, respectively; $p=0.0007)$. Viral load was lower also in seropositive patients, but this was not significant.

\section{Discussion}

We describe a sentinel convenience seroprevalence survey undertaken in the Johannesburg metropolitan area in Gauteng, SA. This survey was designed to complement other sentinel surveys, most notably the study undertaken in Western Cape Province, which mainly sampled patients attending routine antenatal and HIV clinical care. ${ }^{[5]}$ Purposive sampling was conducted to ensure that male patients and

\section{A}
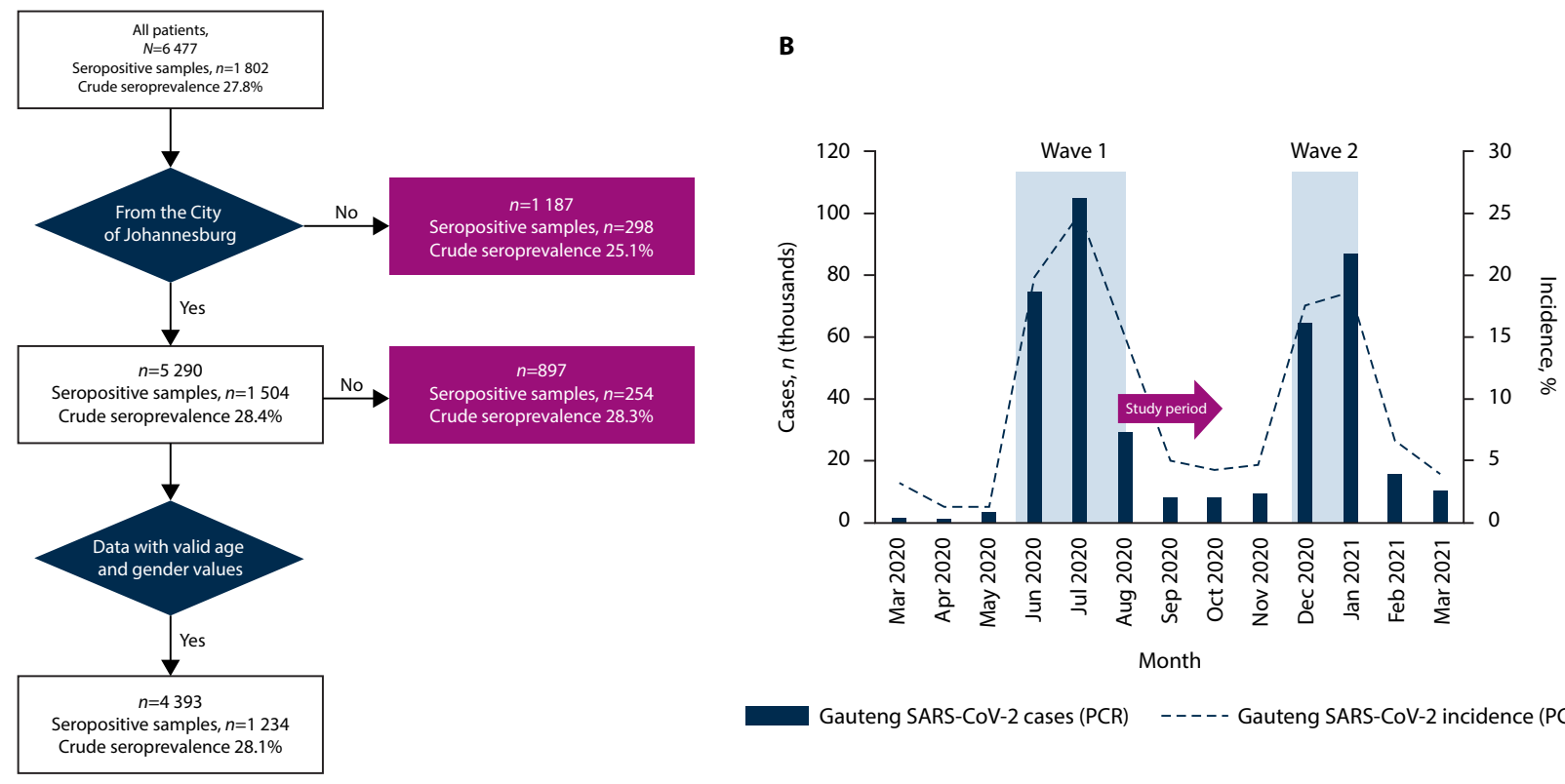

Gauteng SARS-CoV-2 cases (PCR) ----- Gauteng SARS-CoV-2 incidence (PCR)

Fig. 1. (A) Flow diagram for data analysis indicating how data were prepared. (B) Number of cases reported per month between March 2020 and March 2021 diagnosed using SARS-CoV-2 PCR in Gauteng Province, with the incidence reported on the secondary y-axis. (PCR = polymerase chain reaction.) 


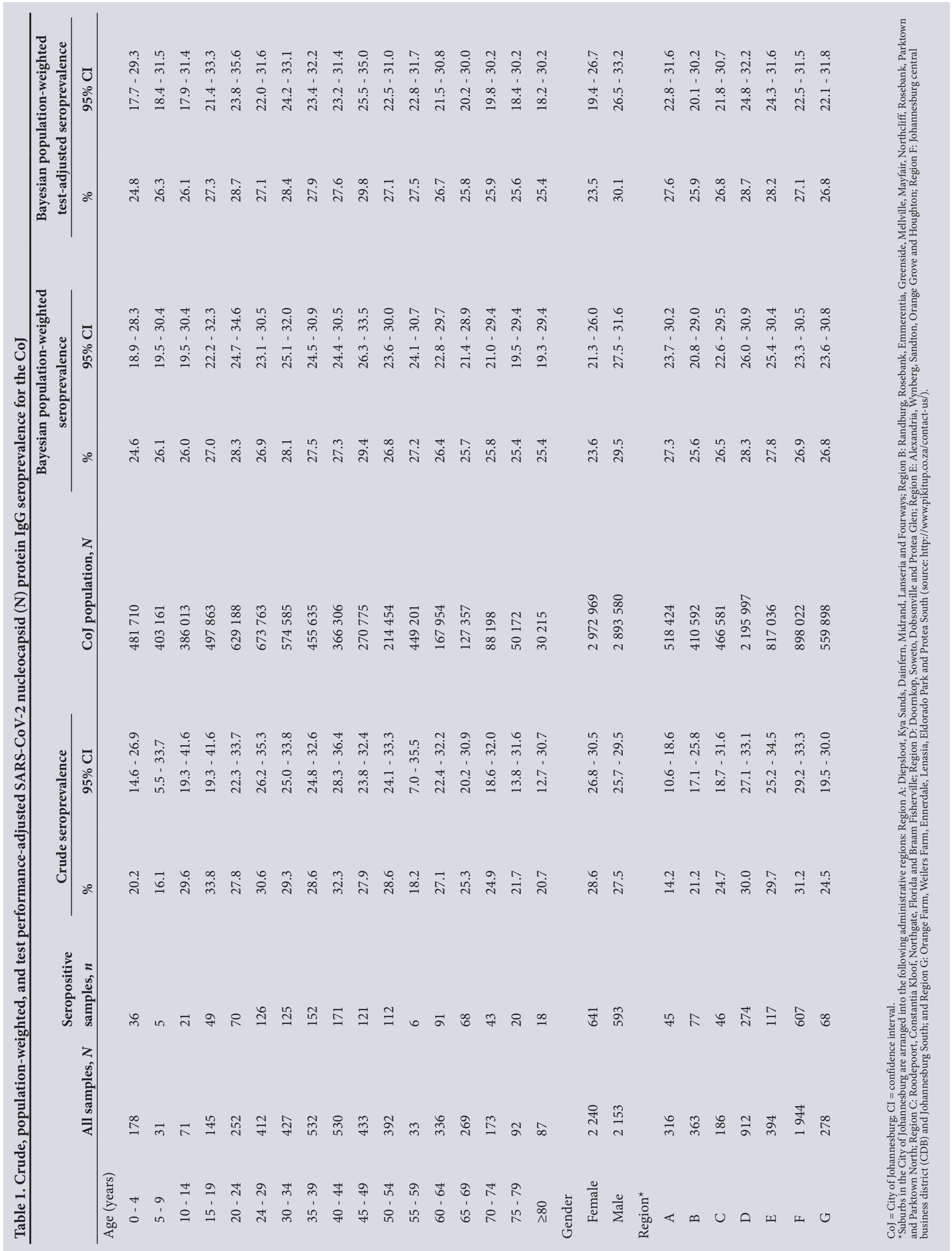


children were included in our study. Overall, the mean age- and testadjusted seroprevalence for SARS-CoV-2 was $27.8 \%$ when averaged across all participants studied, with slightly higher rates for males and peak seropositivity in individuals aged 18 - 49 years. This figure

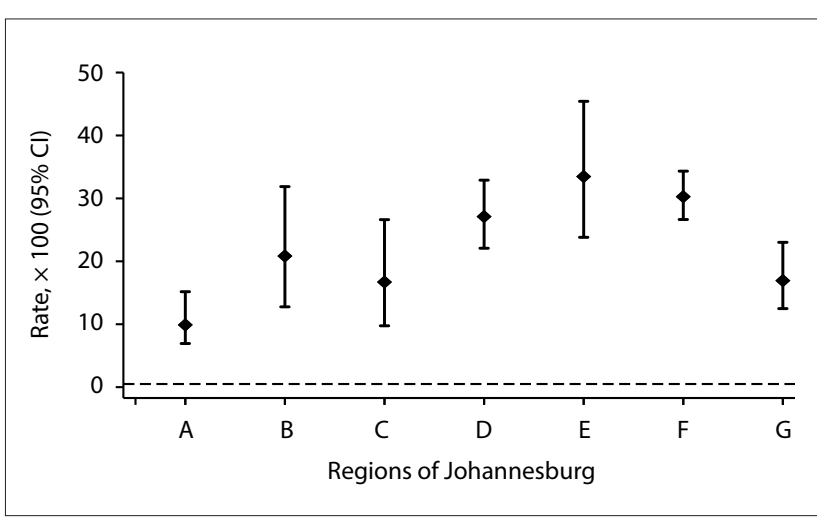

Fig. 2. The SARS-CoV-2 nucleocapsid (N) protein IgG Bayesian populationweighted test-adjusted seroprevalence (reported as rate $\times 100)$ across Johannesburg regions. Suburbs in the City of Johannesburg are arranged into the following administrative regions: Region A: Diepsloot, Kya Sands, Dainfern, Midrand, Lanseria and Fourways; Region B: Randburg, Rosebank, Emmerentia, Greenside, Mellville, Mayfair, Northcliff, Rosebank, Parktown and Parktown North; Region C: Roodepoort, Constantia Kloof, Northgate, Florida and Braam Fisherville; Region D: Doornkop, Soweto, Dobsonville and Protea Glen; Region E: Alexandria, Wynberg, Sandton, Orange Grove and Houghton; Region F: Johannesburg central business district (CDB) and Johannesburg South; and Region G: Orange Farm, Weilers Farm, Ennerdale, Lenasia, Eldorado Park and Protea South (source: http://www.pikitup.co.zal contact-us/). CIs are calculated with the Tiwari et al. ${ }^{[25]}$ formula.

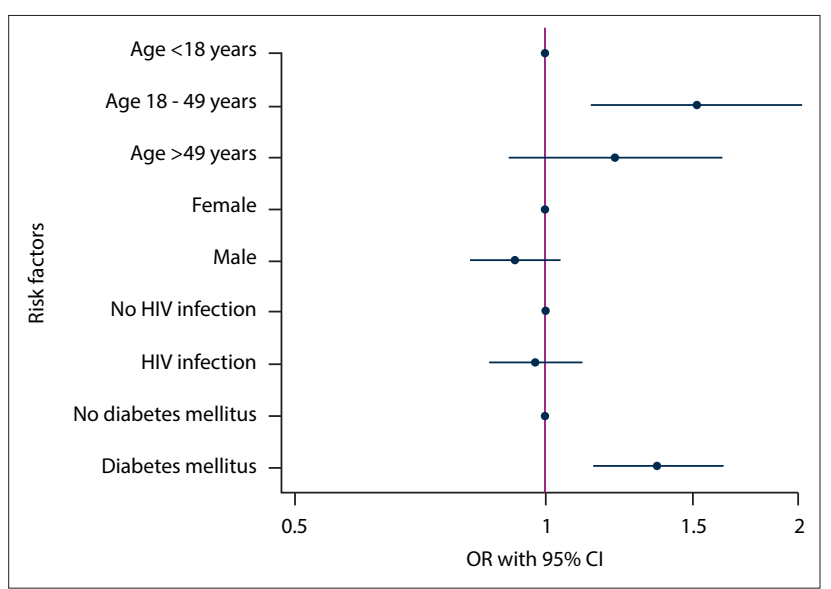

Fig. 3. Forest plot reporting the OR for a positive SARS-CoV-2 nucleocapsid (N) protein IgG test, for various risk factors, between August and October 2020 in Gauteng Province (all sites). (OR = odds ratio; $C I=$ confidence interval.) was lower than the $40 \%$ reported in the Western Cape seroprevalence survey, and may reflect the maturity of the Western Cape epidemic, which peaked earlier than that in Gauteng. In addition, the high numbers of international tourists accessing Cape Town in December 2019 and January 2020 may have resulted in earlier and more widespread seeding than suspected. The figure may also reflect the population sampled. A study conducted among volunteers in the Western Cape showed that seropositivity was higher in people from lower income brackets. ${ }^{[17]}$

The present survey was conducted from August to October 2020. At this time, PCR-confirmed cases of SARS-CoV-2 infection were declining, with the first-wave peak having occurred in July $2020 .^{[5]}$ The timing of this study is important, because detectable serological responses develop at around day 7 and are maximal for IgG after day 14 post symptom onset. Assuming that the presence of IgG antibodies in this study was protective, at least in the short term, the vast majority of those attending hospitals were still immunologically naive. The seroprevalence is expected to increase with subsequent waves, and a recent study of blood donors in SA carried out in January 2021 for predefined days showed a seroprevalence of $60 \%$ among black African donors, with varying figures across provinces. ${ }^{[18]}$ Importantly, our study predates the widespread emergence of the novel 501Y.V2 variant, which in early studies was associated with higher transmissibility. ${ }^{[19]}$

Seropositivity in children was not hugely different to that found in adults. As of January 2021, confirmed COVID-19 cases in those aged $<19$ years were almost six times lower than those in adults, and admissions were even lower. There are limited data on the seroprevalence of COVID-19 antibodies in children. A study from Bavaria reported similar findings to ours in that antibody positivity in children was higher than actual reported cases of COVID-19. ${ }^{[20]}$ This has important public health implications for the debates around guidelines for the reopening of schools.

The age- and sex-adjusted seroprevalence was highest in the more densely populated areas of Johannesburg Inner City, Soweto and surrounds, and Alexandra and surrounds. Overcrowding, often poor sanitation and the impracticability of social distancing, handwashing and mask use are conducive to spread of infection. It has been shown that the COVID-19 caseload, per million population and per $\mathrm{km}^{2}$, is systematically higher in high-density areas compared with lower-density areas. ${ }^{[21]} \mathrm{A}$ limitation of the present study is that we do not have any data from private laboratories, which serve a different, wealthier population.

Patient comorbidities were considered as an individual predictor of antibody production. In HIV-infected individuals for whom data were available, seropositivity correlated significantly with improved immune reconstitution. This finding indicates that HIV-infected individuals are able to produce an antibody response, and may be reassuring in suggesting that those who are well controlled will be appropriate vaccine candidates.

Our findings of increased risk for COVID-19 infection in diabetics as evidenced by the presence of antibodies are in keeping with the literature. ${ }^{[22]}$ Furthermore, diabetics are at increased risk of

Table 2. Trend analysis of hospital admissions and seroprevalence for the $\mathrm{CoJ}^{\star}$

\begin{tabular}{|c|c|c|c|}
\hline Month & Hospital admissions, $N$ & Deaths, $n(\%)$ & Crude seroprevalence, $n / N(\%)$ \\
\hline August 2020 & 1049 & $222(21.2)$ & $419 / 1340(31.3)$ \\
\hline September 2020 & 415 & $69(16.6)$ & $800 / 2883(27.7)$ \\
\hline October 2020 & 256 & $36(14.1)$ & $285 / 1067(26.7)$ \\
\hline
\end{tabular}


severe disease for multiple reasons, including compromised innate immunity and the underlying inflammatory state that predisposes to cytokine storm. COVID-19 infection can also lead to worsening glycaemic control. In SA, only $\sim 60 \%$ of all diabetics are diagnosed and $<10 \%$ are well controlled. ${ }^{[23]}$ We do not have data on the presence of comorbidities in hospitalised patients. However, in view of the interaction between infectious diseases such as tuberculosis, HIV and now COVID-19, it is imperative that increased attention be paid to the detection and management of non-communicable diseases in SA.

\section{Study limitations}

A limitation of the study was that it utilised the Roche Elecsys kit, which detects antibodies produced against the nucleocapsid. These antibodies do not neutralise the virus, and the degree of seroprotection cannot be confirmed. Although recent studies have suggested that nucleocapsid antibodies correlate with levels of neutralising antibodies, this represents an important caveat of this study. ${ }^{[24]}$ However, the detection of past infection in these individuals will be important in assessing the effectiveness of public health and non-pharmacological control methods in SA patients and will assist in informing the vaccine roll-out. In addition, it may provide information regarding the potential implications of new variant mutations in SA, which may have increased infectivity potential. Furthermore, we used remnant samples from patients attending hospitals or clinics. Some of these patients may have attended for COVID-19-related reasons, and we may therefore have overestimated community-level seroprevalence. As we did not have clinical details, it is possible that the association of higher CD4 T-cell counts in seropositive individuals may represent early HIV infection.

\section{Conclusions}

Our estimates of seroprevalence, carried out during the first wave of the pandemic, are slightly lower than a similar sentinel survey from the Western Cape. We showed that almost one in three people in the CoJ were infected during the first wave of the COVID-19 pandemic. Combined with household surveys, these results can be used to determine the infection fatality risk, which is a measure of the severity of infection. Prevalence estimates are crucial for weighing the risks and benefits of future strategies for the next anticipated waves of infections. Our findings of high seroprevalences in children, in high-density areas and in diabetics have important public health implications. The high seroprevalence in people with HIV infection portends well for vaccination in this group.

\section{Declaration. None.}

Acknowledgements. We thank the laboratories that provided the remnant samples for our study. We also thank the laboratory staff for conducting the testing and the Division of Public Health Surveillance and Response at the National Institute for Communicable Diseases for providing aggregate PCR and DATCOV data.

Author contributions. JAG: supervision, project administration, writing of first draft. JAG, IM and NC: formal analysis and visualisation. All authors: conceptualisation, writing - original draft preparation, writing review, editing, and methodology and resources.
Funding. None.

Conflicts of interest. None.

1. Zhu N, Zhang D, Wang W, et al. A novel coronavirus from patients with pneumonia in China, 2019.

N Engl J Med 2020;382(8):727-733. https://doi.org/10.1056/NEJMoa2001017
2. World Health Organization. Director-General's opening remarks at the media briefing on COVID-19 2. World Health Organization. Director-General's opening remarks at the media briefing on COVID-19-
11 March 2020. 11 March 2020. https://www.who.int/director-general/speeches/detail/who-directorgeneral-s-opening-remarks-at-the-media-briefing-on-covid-19---11-march-2020 (accessed 10 January 2021).

3. National Institute for Communicable Diseases. First case of COVID-19 announced - an update. 5 March 2020. https://www.nicd.ac.za/first-case-of-covid-19-announced-an-update/ (accessed 12 December 2020).

4. Abdool Karim SS. The South African response to the pandemic. N Engl J Med 2020;382(24):e95. https://doi.org/10.1056/NEJMc2014960

5. National Institute for Communicable Diseases. Laboratory confirmed cases of COVID-19 in National Institute for Communicable Diseases. Laboratory confirmed cases of COVID-19 in
South Africa. Week 30, 2020. https://www.nicd.ac.za/wp-content/uploads/2020/07/NICD-WeeklyEpidemiological-Brief_-Week-ending-25-July-2020.pdf (accessed 10 January 2021).

6. World Health Organization. WHO Coronavirus Disease (COVID-19) Dashboard. https://covid19. who.int/region/afro/country/za (accessed 10 January 2021).

7. Centre for Respiratory Diseases and Meningitis and Outbreak Response Unit, Division of Public Health Surveillance and Response, National Institute for Communicable Diseases, and National Department of Health, South Africa. Coronavirus disease 2019 (COVID-19) caused by a novel coronavirus (SARSCoV-2): Guidelines for case-finding, diagnosis, management and public response. 3 July 2020. https:// www.nicd.ac.za/wp-content/uploads/2020/07/NICD_DoH-COVID-19-Guidelines_Final_3-Jul-2020, www (accessed 10 December 2020).

8. Rostami A, Sepidarkish M, Leeflang MMG, et al. SARS-CoV-2 seroprevalence worldwide: A systematic review and meta-analysis. Clin Microbiol Infect 2021;27(3):331-340. https://doi.org/10.1016/j. cmi.2020.10.020

9. Chibwana MG, Jere KC, Kamngona R, et al. High SARS-CoV-2 seroprevalence in health care workers but relatively low numbers of deaths in urban Malawi. medRxiv 2020 (epub 1 August 2020). https:// doi.org/10.1101/2020.07.30.20164970

10. Uyoga S, Adetifa IMO, Karanja HK, et al. Seroprevalence of anti-SARS-CoV-2 IgG antibodies in Kenyan blood donors. Science 2021;371(6524):79-82. https://doi.org/10.1126/science.abe1916

11. Hsiao M, Davies M-A, Kalk E, et al. SARS-CoV-2 seroprevalence in the Cape Town metropolitan sub-districts after the peak of infections. National Institute for Communicable Diseases, COVID-19 Special Public Health Surveillance Bulletin, 28 September 2020. https://www.nicd.ac.za/wp-content/ uploads/2020/09/COVID-19-Special-Public-Health-Surveillance-Bulletin_Issue-5.pdf (accessed 8 February 2021).

12. Gouda HN, Charlson F, Sorsdahl K, et al. Burden of non-communicable diseases in sub-Saharan Africa, 1990 - 2017: Results from the Global Burden of Disease Study 2017. Lancet Glob Health 2019; 7(10):e1375-e1387. https://doi.org/10.1016/S2214-109X(19)30374-2

13. Yang J, Zheng Y, Gou X, et al. Prevalence of comorbidities and its effects in patients infected with SARS-CoV-2: A systematic review and meta-analysis. Int J Infect Dis 2020;94:91-95. https://doi. org/10.1016/j.jiji.2020.03.017

14. Grove J, Mayne E, Burgers W, et al. Validation of Roche immunoassay for severe acute respiratory coronavirus 2 in South Africa. South Afr J Infect Dis 2021;36(1):a286. https://doi.org/10.4102/sajid. v36i1.286

15. Mahase E. Covid-19: Two antibody tests are 'highly specific' but vary in sensitivity, evaluations find. BMJ 2020;369:m2066. https://doi.org/10.1136/bmj.m2066

16. National SARS-CoV-2 Serology Assay Evaluation Group. Performance characteristics of five immunoassays for SARS-CoV-2: A head-to-head benchmark comparison. Lancet Infect Dis 2020;20(12):1390-1400. https://doi.org/10.1016/S1473-3099(20)30634-4

17. Shaw JA, Meiring M, Cummins T, et al. Higher SARS-CoV-2 seroprevalence in workers with lower socioeconomic status in Cape Town, South Africa. PLoS ONE 2021;16(2):e0247852. https://doi. org/10.1371/journal.pone. 0247852

18. Sykes W, Mhlanga L, Swanevelder R, et al. Prevalence of anti-SARS-CoV-2 antibodies among blood donors in Northern Cape, KwaZulu-Natal, Eastern Cape, and Free State provinces of South Africa in January 2021. Res Square 2021:rs.3.rs-233375. https://doi.org/10.21203/rs.3.rs-233375/v1

19. National Institute for Communicable Diseases. Dominance of the SARS-CoV-2 501Y.V2 lineage in Gauteng. 28 January 2021. https://www.nicd.ac.za/wp-content/uploads/2021/01/Dominance-of-theSARS-CoV-2-501Y.V2-lineage-in-Gauteng-South-Africa-1.pdf (accessed 2 March 2021).

20. Hippich M, Holthaus L, Assfalg R, et al. A public health antibody screening indicates a 6 -fold higher SARS-CoV-2 exposure rate than reported cases in children. Med (NY) 2021;2(2):149-163.e4. https:// doi.org/10.1016/j.medj.2020.10.003

21. Sahasranaman A, Jensen HJ. Spread of COVID-19 in urban neighbourhoods and slums of the developing world. J R Soc Interface 2021;18:20200599. https://doi.org/10.1098/rsif.2020.0599

22. Singh AK, Gupta R, Ghosh A, Misra A. Diabetes in COVID-19: Prevalence, pathophysiology, prognosis and practical considerations. Diabetes Metab Syndr 2020;14(4):303-310. https://doi. org/10.1016/j.dsx.2020.04.004

23. Mutyambizi C, Booysen F, Stokes A, Pavlova M, Groot W. Lifestyle and socio-economic inequalities in diabetes prevalence in South Africa: A decomposition analysis. PLoS ONE 2019;14(1):e0211208. https://doi.org/10.1371/journal.pone.0211208

24. Suhandynata RT, Hoffman MA, Huang D, et al. Commercial serology assays predict neutralisation activity against SARS-CoV-2. Clin Chem 2020;67(2):404-414. https://doi.org/10.1093/clinchem/ hvaa262

25. Tiwari RC, Clegg LX, Zou Z. Efficient interval estimation for age-adjusted cancer rates. Stat Methods Med Res 2006;15(6):547-569. https://doi.org/10.1177/0962280206070621 